\title{
Trombose venosa profunda como complicação da escleroterapia química no tratamento de telangiectasias dos membros inferiores
}

\author{
D eep venous thrombosis as complication of chemical sclerotherapy \\ in the treatment of leg telangiectasias
}

\author{
Adilson Ferraz Paschôa', Luciana H ayashida², M arcelo Kurz Siqueira², Bonno van Bellen ${ }^{3}$
}

\begin{abstract}
Resumo
O s autores relatam dois casos de escleroterapia de telangiectasias, as quais complicaram com trombose venosa profunda. 0 primeiro caso foi confirmado por flebografia, e o segundo, por duplex scan. U m paciente, 8 anos após, apresentou uma tromboflebite espontânea de veia safena parva, que resultou em pesquisa de trombofilia positiva para o Fator V Leiden. A outra paciente teve pesquisa de trombofilia negativa. Os relatos de tromboembolismo relacionado à escleroterapia são escassos na literatura. 0 objetivo do trabal ho é alertar para essa possibilidade, valorizan do as queixas de dor e edema após a escleroterapia. $\mathrm{H}$ avendo suspeita clínica, o duplex scan deve ser realizado.
\end{abstract}

Palavras-chave: trombose de veias profundas, escleroterapia, complicações.

A prática da escleroterapia química para o tratamento das varizes dos membros inferiores remonta à metade do século XIX, com a utilização de álcool absoluto, aparentementetrazendo bonsresultados, mas com alto índice de complicações e mortalidade por sepse e fenômenos embólicos ${ }^{1}$. Beniamino Schiassi, cirurgião de Bologna, descreveu, em 1908, a injeção de uma solução de iodo em uma veia previamente disseca-

1. Mestrando pela Universidade Estadual de Campinas (UNICAMP). Cirurgião vascular, Serviço de Cirurgia Vascular e Angiologia, Hospital da Beneficência Portuguesa de São Paulo, SP.

2. Pós-graduando(a) do Serviço de Cirurgia Vascular e Angiologia, Hospital da Beneficência Portuguesa de São Paulo, SP.

3. Livre-docente. Cirurgião vascular e chefe do Serviço de Cirurgia Vascular e Angiologia, Hospital da Beneficência Portuguesa de São Paulo, SP.

Artigo submetido em 22.08.05, aceito em 28.09.05.

Copyright $\odot 2005$ by Sociedade Brasileira de Angiologia e Cirurgia Vascular.

\begin{abstract}
The authors report two cases of sclerotherapy for telangiectasias, which complicated with deep venous thrombosis. The first case was confirmed by phlebography and the second one by duplex scan. 0 ne patient, 8 years later, had a spontaneous lesser saphenous vein thrombophlebitis, which resulted in positive thrombophilia investigation for factor $\mathrm{V}$ Leiden. The other patient had negative investigation for thrombophilia. There are very few reports on thromboembolism after sclerotherapy in the literature. Thisstudy aims to warn against this possibility, valuing the complaints of pain and swollen leg after the sclerotherapy. In case of clinical suspicion, a duplex scan should be performed.
\end{abstract}

Key words: deep venous thrombosis, sclerotherapy, complications.

da como tratamento complementar à cirurgia. No entanto, em razão de sua natureza, tal solução possuía efeitos imprevisíveis. As tentativas do químico Paul Ehrlich de encontrar um composto químico apropriado levaram o professor Sicard a introduzir o primeiro "esclerosante seguro", o salicilato de sódio, em 1911. Ao longo do século XX, a utilização da escleroterapia ganhou vários adeptos e teve períodos alternados de menor ou maior aplicação².

A despeito da evolução tecnológica que abriu espaço para outras modalidades, como a laserterapia, a escleroterapia química ou convencional mantém o seu lugar de opção preferencial devido aos melhores resultados terapêuticos e menor custo de aplicação.

$\mathrm{N}$ ão obstante o reconhecido emprego da escleroterapia para o tratamento das varizes tronculares ecolaterais, associada ou não à cirurgia convencional, dentro 
das classes II e superiores da classificação CEAP3, a experiência brasileira é muito diferente. 0 Brasil é um país tropical, com áreas geográficas ensolaradas até 300 dias por ano. Essa característica climática, associada a aspectoscomportamentais, gerauma preocupação estética altamente valorizada. Portanto, desenvolvemos enorme experiência no tratamento escleroterápico de telangiectasia e veias reticulares, incluídas dentro da categoria CEAP I.

A escleroterapia, como qualquer modalidade terapêutica invasiva, apresenta um potencial para reações adversas e complicações. Acredita-se que as taxas de complicação para esse procedimento sejam baixas, sendo a hiperpigmentação e a úlcera de pele as mais temidas, já que implicam em mau resultado estético 4,5 . A associação da escleroterapia com a ocorrência de tromboembolismo venoso tem citações escassas na literatura6-8. Livros-texto também citam essa possibilidade, mas a fal ta de reprodução de citações bibliográficas sugere que as informações sejam fruto de experiência pessoal dos autores. 0 objetivo deste trabal ho é relatar a ocorrência de dois casos de tromboembolismo venoso que se manifestaram após sessão de escleroterapia química com solução hipertônica de glicose a 75\%.

\section{Relato dos casos}

C aso 1 - R.F.B., 34 anos, portadora de telangiectasias classe CEAP I, em 1994 foi submetida, por opção estética, a escleroterapia com solução hipertônica de glicosea $75 \%$. U ma sessão deescleroterapiaquímica em nosso serviço consiste em 15 a 20 injeções com consumo de 3 a $5 \mathrm{ml}$ de substância esclerosante. Após 5 dias, iniciou-sequadro dedor eedema depanturrilha esquerda, com dificuldade para apoiar o pé no chão. Submetida a flebografia ascendente, detectou-se trombose venosa acometendo parcialmente a veia poplítea esquerda distal. A pacientefoi tratada com heparina nãofracionada por 7 dias eadministração concomitante de varfarina sódica. A anticoagulação oral foi mantida por 6 meses, controlando-se o tempo de protrombina dentro da faixa de IN R entre 2 e 3 . A paciente foi desaconselhada a continuar o uso de pílula anticoncepcional, que fazia por 6 anos seguidos.

Após8 anos, a pacienteretornou com queixa dedor em região posterior da perna direita. Tinha realizado viagem aérea de 6 horas de duração 2 dias antes do aparecimento da dor. No exame clínico, havia dor à palpação da panturrilha e cordão indurado na região posterior da perna. A impressão clínica de tromboflebite superficial de veia safena parva foi confirmada pelo duplex scan. A paciente foi tratada com heparina de baixo peso molecular em dose terapêutica subcutânea (enoxaparina sódica $1 \mathrm{mg} / \mathrm{kg}$ de12/12h) por período de 7 dias, associada à compressão elástica e medicação antiinflamatória. O ptou-se por não administrar a profilaxia secundária, considerando-se que havia fator de risco bem definido relacionado à ocorrência da trombose. H ouveresolução completa do processo inflamatório em 3 semanas.

D iante do episódio recorrente, a paciente foi submetida a pesquisa de trombofilia (proteína $C$, proteína $S$, antitrombina, fator $V$ Leiden, mutação do gen da protrombina, homocisteína, anticorpos antifosfolípides), a qual acusou a presença do Fator $V$ Leiden em heterozigoze.

Caso 2 - R.A.G., 23 anos, portadora de telangiectasias em ambos os membros inferiores. Fazia uso de pílula anticoncepcional há 6 meses para controle de disfunção hormonal. Por opção estética, iniciou-se tratamento de escleroterapia com solução hipertônica de glicose a $75 \%$, com intervalo quinzenal entre as sessões. As sessões foram conduzidas da mesma forma descrita no relato anterior.

U m dia após a terceira sessão, queixou-se de dor e inchaço em região póstero-lateral da perna esquerda e dificuldade para deambulação. N o exameclínico, havia edema moderado e dor intensa à palpação local. 0 duplex scan confirmou trombose de veias fibulares atéa confluência com a veia poplítea. A paciente, após assinar o termo de consentimento informado, foi randomizada para integrar um estudo de esquematerapêutico com droga inibidora indireta da trombina. A pesquisa de trombofilia mostrou-se negativa.

\section{D iscussão}

As informações sobre a ocorrência do tromboembolismo venoso associado à escleroterapia detelangiectasias eveias reticulares da classe C EAP I são escassas na literatura. Relatos demeados do século passado indicavam baixíssima incidência de tromboembolismo ${ }^{9,10}$, embora fossem referentes à escleroterapia associada à compressão elástica para o tratamento de veias varicosas. T odavia, a maioria dessas informações era obtida a partir da impressão clínica e, portanto, imprecisas, se considerarmos a natureza silenciosa de boa parte dos fenômenos tromboembólicos. 
O diagnóstico clínico da trombose venosa profunda subestima a real ocorrência em, pelo menos, $50 \%$ dos casos, se comparado com a técnica do fibrinogênio marcado, a flebografia e, modernamente, ao duplex scan ${ }^{11}$.

Williams \& W ilson ${ }^{12}$ estudaram, com pletismografia de impedância e D oppler ultra-som, 67 membros em 50 pacientes submetidos a escleroterapia com compressão associada, pela técnica de Fegan. As avaliações feitas 1 e 2 semanas após o procedimento não revelaram qualquer alteração sugestiva da ocorrência de trombose pelos métodos utilizados. T odavia, os autores reconheciam que ambos os exames realizados não se comparavam à flebografia. Esse trabalho representa uma época anterior ao uso sistemático do duplex scan.

Böhler-Sommeregger et al. ${ }^{13}$ investigaram 15 pacientes com flebografia ascendente associada à injeção concomitante de solução radiopaca diretamente nas telangiectasias. O bservaram, em dois pacientes, a passagem do meio de contraste para as veias femorais. Concluíram que determinadas condições anatômicas poderiam aumentar o risco de trombose venosa iatrogênica. Considerando a natureza multifatorial do tromboembolismo venoso ${ }^{14}$, tais condições anatômicas deveriam somar-sea outrosfatores deordem genética ou ambiental para compor um quadro favorável à instalação da "T ríade de Virchow".

N o primeiro caso relatado, a evolução do conhecimento acumulado nos últimos 10 anos do estudo da trombofilia culminou com o diagnóstico do Fator $\mathrm{V}$ Leiden ${ }^{15}$. Essa mutação acomete $2 \%$ da população geral brasileira e de 6 a $20 \%$ dos doentes portadores de tromboembolismo venoso em nosso meio ${ }^{16,17}$

$\mathrm{N}$ a ocasião em que essa doente desenvolveu 0 quadro de trombose venosa, fazia uso de medicação anticoncepcional, a qual foi abandonada após o diagnóstico. $\mathrm{N}$ o diagnóstico do primei ro evento trombótico, foi utilizada a flebografia ascendente, pois nessa época não havia disponibilidade do duplex scan em nosso serviço. N esse caso, a escleroterapia deve ter desempenhado o papel de risco temporal, o qual, na história futura da mesma paciente, foi substituído por uma viagem aérea prolongada. O utro fato a considerar é a importância da idade para a redução do limiar trombótico, uma vez que 0 aumento da idadeé reconhecido como fator de risco independente para trombose venosa ${ }^{18}$.
No segundo caso descrito, já contando com a experiência do primeiro e considerando que se tratava de uma paciente jovem em uso de estrógeno para a regulação menstrual, optou-se pela pesquisa de trombofilia. 0 estudo não revelou qualquer alteração genética ou adquirida.

A investigação indiscriminada defatorestrombofílicos em clientes candidatas ao tratamento deescleroterapia não é justificável. O utras iniciativas similares, como as pesquisas de trombofilia em pacientes que vão iniciar a anticoncepção hormonal, não se mostraram eficientes, considerando o grande contingente de exames negativos necessários para se evitar a trombose venosa proximal ou a embolia pulmonar fatal 19,20 . Todavia, a anamnese detalhada pode revelar pontos importantes, principalmente na história pregressa e familiar, que possam motivar a investigação laboratorial em casos selecionados.

R essaltamos que a prática da escleroterapia, mesmo direcionada para pequenas telangiectasias com menos de $1 \mathrm{~mm}$ de diâmetro, pode gerar complicações importantes. Possivelmente, se levarmos em conta a quantidade de pacientes tratados, o número absoluto de doentes que desenvolvem o tromboembolismo venoso seja considerável, e parte deles não diagnosticados, seja por falta de quadro clínico evidente, de recursos técnicos para confirmação diagnóstica ou de interesse em compreender a queixa do paciente, muitas vezes atenuada pela prática da automedicação, como complicação da escleroterapia.

0 objetivo deste relato foi chamar a atenção para a comunidade de cirurgiões vasculares, muitos com grande experiência no campo da escleroterapia estética, a estarem atentos para a possibilidade do tromboembolismo venoso e não negligenciarem as queixas dos pacientes, principal mente nos primeiros dias que seguem ao tratamento. H avendo dúvida diagnóstica, o duplex scan deverá ser sempre realizado.

\section{R eferências}

1. Schneider W. Contribution to the history of the sclerosing treatment of varicesand to itsanatomo-pathologic study. Bull Soc Fr Phlebol. 1965;18:117-30.

2. Goldman M P. H istorical aspects of treatment. In: Goldman $M P$, editor. Treatment of varicose and teleangiectatic leg veins. M osby; 1995. p. 1-10.

3. Porter JM, M oneta $G L$ and an International Consensus Committee on Chronic V enous D isease. Reporting standards in venous disease: an update. J V asc Surg. 1995;21:635-45. 
4. Goldman M P, Bennett RG. Treatment of telangiectasia: a review. J Am Acad D ermatol. 1987;17:167-82.

5. W eiss RA, W eiss M A, Goldman M P. Physicians' negative perception of sclerotherapy for venous disorders: review of a 7year experience with modern sclerotherapy. South $\mathrm{M}$ ed J. 1992;85:1101-6.

6. Smith $F L$, Johnson $M A$. Incidence of pulmonary embolism after venous sclerosing therapy. M inn M ed J. 1948;31:270.

7. Guex, JJ. Thrombotic complications of varicose veins: a literature review of the role of superficial venous thrombosis. D ermatol Surg. 1996;22:378-82.

8. Yamaki T, N ozaki M, Sasaki K. Acute massive pulmonary embolism following high ligation combined with compression sclerotherapy for varicose veins. Dermatol Surg. 1999;25: 321-5.

9. FeganW G. Continuous compression technique of injecting varicose veins. Lancet. 1963;2:109-12.

10. Beresford SA, Chant $A D$, Jones H O, Piachaud D. V aricose veins: a comparison of surgery and injection/compression sclerotherapy. Lancet. 1978;1:921-4.

11. Kakkar VV. D eep vein thrombosis of the leg. Am J Surg. 1970;120:527.

12. W illiams RA, Wilson SE. Sclerosant treatment of varicose veinsand deep vein thrombosis. Arch Surg. 1984;119:1283-5.

13. Bohler-Sommeregger K, K arnel F, Schuller-Petrovic S, Santler $R$. Do teleangiectases communicate with the deep venous system? J D ermatol Surg O ncol. 1992;18:403-6.

14. Rosendaal FR. Venous thrombosis: a multicausal disease. Lancet. 1999;353:1167-73.
15. Bertina RM , Koeleman B, Koster T, et al. M utation in blood coagulation factor $\mathrm{V}$ associated with resistance to activated protein C. N ature. 1994;369:64-7.

16. ArrudaVR, Annichino-Bizzacchi JM , CostaFF, ReistmaPH . Factor V Leiden (FVQ 506) is common in a Brazilian population. Am J H ematol. 1995;49:242-3.

17. Franco RF, Reitsma PH, Lourenço D, et al. Factor XIII $V$ al34L eu is a genetic factor involved in the etiology of venous thrombosis .T hrom H aemost. 1999;81:676-9.

18. Ridker PM , Glynn RJ, M iletich JP, G oldhaber SZ, Stampfer $\mathrm{MJ}, \mathrm{H}$ ennekens $\mathrm{CH}$. Age-specific incidence rates of venous thromboembolism among heterozygous carriers of factor $\mathrm{V}$ Leiden mutation. Ann Intern M ed. 1997;126:528-31.

19. Vandenbroucke JP, van der $M$ eer FJ, Helmerhorst FM, Rosendaal FR. Factor V Leiden: should we screen oral contraceptive users and pregnant women? BM J. 1996;313: 1127-30.

20. R osendaal FR. O ral contraceptives and screening for factor $V$ Leiden. Thromb H aemost. 1996;75:524-5.

\section{Correspondência:}

Adilson Ferraz Paschôa

Rua $M$ anuel Jacinto, 932/34 Bloco 11

CEP 05624-001 - São Paulo, SP

E-mail: adilsonpaschoa@uol.com.br

\section{Aviso aos ex-residentes}

Se você foi residente até 2004 e ainda não está inscrito em sua R egional, procure regularizar sua situação para passar a receber $0 \mathbf{J}$ Vasc $\mathbf{B r}$ imediatamente, sem qualquer ônus.

Ajude a manter o cadastro da SBACV atualizado. 